\title{
The Effects of Genotype $\times$ Phenotype Interactions on Transcriptional Response to Silver Nanoparticle Toxicity in Organotypic Cultures of Murine Tracheal Epithelial Cells
}

\author{
Tyler P. Nicholas (1), ${ }^{*, \dagger}$ Anoria K. Haick, ${ }^{\dagger}$ Theo K. Bammler, ${ }^{*}$ \\ Tomomi W. Workman, ${ }^{*}$ Terrance J. Kavanagh, ${ }^{, \dagger}$ Elaine M. Faustman (๑), ${ }^{*}$ \\ Sina A. Gharib, ${ }^{\dagger, 1}$ and William A. Altemeier ${ }^{\dagger, 1,2}$
}

${ }^{*}$ Department of Environmental and Occupational Health Sciences; and ${ }^{\dagger}$ Division of Pulmonary, Critical Care, and Sleep Medicine, Department of Medicine, University of Washington, Seattle, Washington

${ }^{1}$ Co-senior authors.

${ }^{2}$ To whom correspondence should be addressed at UW Medicine Research/South Lake Union, 850 Republican Street, S324, Box 358052, Seattle, WA 98109. Fax: (206) 221-0739. E-mail: billa@uw.edu.

\begin{abstract}
The airway epithelium is critical for maintaining innate and adaptive immune responses, and occupational exposures that disrupt its immune homeostasis may initiate and amplify airway inflammation. In our previous study, we demonstrated that silver nanoparticles (AgNP), which are engineered nanomaterials used in multiple applications but primarily in the manufacturing of many antimicrobial products, induce toxicity in organotypic cultures derived from murine tracheal epithelial cells (MTEC), and those differentiated toward a “Type 2 [T2]-Skewed" phenotype experienced an increased sensitivity to AgNP toxicity, suggesting that asthmatics could be a sensitive population to AgNP exposures in occupational settings. However, the mechanistic basis for this genotype $\times$ phenotype $(G \times P)$ interaction has yet to be defined. In this study, we conducted transcriptional profiling using RNA-sequencing to predict the enrichment of specific canonical pathways and upstream transcriptional regulators to assist in defining a mechanistic basis for $\mathrm{G} \times \mathrm{P}$ effects on AgNP toxicity. Organotypic cultures were derived from MTEC across 2 genetically inbred mouse strains (A/J and C57BL/6J mice), 2 phenotypes ("Normal" and "T2-Skewed"), and 1 AgNP exposure (an acute 24 h exposure) to characterize G $\times$ P effects on transcriptional response to AgNP toxicity. The "T2-Skewed" phenotype was marked by increased pro-inflammatory T17 responses to AgNP toxicity, which are significant predictors of neutrophilic/difficult-to-control asthma and suggests that asthmatics could be a sensitive population to AgNP exposures in occupational settings. This study highlights the importance of considering $\mathrm{G} \times \mathrm{P}$ effects when identifying these sensitive populations, whose underlying genetics or diseases could directly modify their response to AgNP exposures.
\end{abstract}

Key words: silver nanoparticles; gene-environment interactions; organotypic cultures; airway epithelial cells; inflammation.

Upstream perturbations of canonical inflammatory pathways within the respiratory system can lead to adverse cellular responses, such as inflammation, which can profoundly impact the ability of exposed airways to maintain cellular function and overall health. There is presently a need for the development of in vitro models to provide relevant data on risk assessment for 
occupational exposures. Despite their economic advantages over in vivo models, in vitro models are currently limited by: (1) the use of immortalized or transformed cell lines that may not recapitulate primary cell phenotypes, (2) the use of cells from a single genetic background or phenotype, which may not capture the impact of underlying genetics or diseases on toxicant response, and (3) the limited efforts to relate in vitro exposures to occupational exposures to provide a mechanistic basis for sensitivity to occupational exposures and inform regulatory policy aimed at protecting all populations (Judson et al., 2011; Krewski et al., 2009).

Silver nanoparticles (AgNP) have been tested for respiratory toxicity in previous studies, as they are used in the manufacturing of many antimicrobial consumer products, including air filters, humidifiers, and purifiers as well as antimicrobial sprays (Quadros and Marr, 2010, 2011; Vance et al., 2015). Previous in vitro studies observed the mode of action (MoA) for their antimicrobial properties (eg, release of bioactive silver ions $\left[\mathrm{Ag}^{+}\right]$ upon dissolution; Danilczuk et al., 2006; Kim et al., 2007; Ma et al., 2012) is also a defining factor of AgNP toxicity in mammalian cells (Yamanaka et al., 2018). One MoA in airway epithelial cells is $\mathrm{Ag}^{+}$-mediated reactive oxygen species (ROS) production, which leads to adverse cellular responses including oxidative stress, mitochondrial dysfunction, inflammation, and cytotoxicity (Choo et al., 2016; Cronholm et al., 2013; Gliga et al., 2014; Jeannet et al., 2015a,b; Kim et al., 2011, 2014; Lerner et al., 2016; Schlinkert et al., 2015; Zhang et al., 2015). Cytokines are critical mediators of asthma, and regulate this heterogeneous chronic airway disease through divergent mechanisms of type 2 (T2) and T17 inflammation to produce the distinct endotypes of increasing severity, T2/T17-low, T2-high, and T17-high (Choy et al., 2015). The T2-high endotype is associated with eosinophilic/easy-to-control asthma (Choy et al., 2011; Woodruff et al., 2009), whereas the T17-high endotype is associated with neutrophilic/difficult-to-control asthma, and is thus characterized by the secretion of potent neutrophil-attractant chemokines (Colobran et al., 2007; Commins et al., 2010). Asthmatics are possibly a sensitive population to AgNP exposures in occupational settings, as these adverse cellular responses may further modulate innate and adaptive immune responses at the epithelial-immune interface to exacerbate this chronic airway disease by shifting it toward a T17-high endotype (Alsaleh and Brown, 2018).

We previously characterized the effects of interactions between host genetic and acquired factors, or gene $\times$ environment $(G \times E)$ interactions, on AgNP toxicity (Nicholas et al., 2019). Understanding $\mathrm{G} \times \mathrm{E}$ effects is important for identifying sensitive populations, whose underlying genetics or diseases could directly modify their response to AgNP exposures. This study was designed to test the hypothesis that genotype and phenotype (physiological environment) will define $\mathrm{G} \times \mathrm{E}$, or genotype $\times$ phenotype $(G \times P)$ interaction, effects on transcriptional response to AgNP toxicity. We derived organotypic cultures from primary murine tracheal epithelial cells (MTEC) of differentially sensitive genotypes (A/J and C57BL/6J mice) to airway hyperresponsiveness (AHR) (Ackerman et al., 2005; Karp et al., 2000; Levitt et al., 1990), antigen sensitization (Brewer et al., 1999), T2 lung inflammation (Shinagawa and Kojima, 2003), and engineered nanomaterial toxicity (Scoville et al., 2015, 2017) to model the effects of host genetic factors on AgNP toxicity. We differentiated organotypic cultures toward either "Normal" or "T2Skewed" phenotypes to model the effects of host acquired factors on AgNP toxicity, as previously described in Nicholas et al. (2019).
Using this high-content in vitro model of the conducting airway, we characterized global, differential, and targeted gene expression, canonical pathway enrichment, and upstream transcriptional regulation to understand $G \times P$ effects on transcriptional response to AgNP toxicity. To our knowledge, this is the first study to use organotypic cultures as a high-content in vitro model of the conducting airway paired with RNA-sequencing (RNA-seq) to characterize $\mathrm{G} \times \mathrm{P}$ effects on transcriptional response to AgNP toxicity. By pairing organotypic cultures with RNA-seq, we can capture the enrichment of specific canonical pathways and identify their transcriptional regulators to assist in defining a mechanistic basis for $\mathrm{G} \times \mathrm{P}$ effects on AgNP toxicity.

\section{MATERIALS AND METHODS}

Cell culture. All animal studies were approved by the Institutional Animal Care and Use Committee at the University of Washington. We harvested tracheas from A/J and C57BL/6J mice (The Jackson Laboratory, Bar Harbor, Maine), and isolated MTEC using enzymatic digestion, as previously described in Horani et al. (2013), You and Brody (2013), and You et al. (2002). We suspended MTEC in a defined proliferation media (Dulbecco's Modified Eagle Media [DMEM] with $10 \mu \mathrm{g} / \mathrm{ml}$ insulin, $5 \mu \mathrm{g} / \mathrm{ml}$ apo-transferrin, $0.1 \mu \mathrm{g} / \mathrm{ml}$ cholera toxin, $25 \mathrm{ng} / \mathrm{ml}$ epidermal growth factor, $30 \mu \mathrm{g} / \mathrm{ml}$ bovine pituitary extract, and 50 $\mathrm{nM}$ retinoic acid [Sigma-Aldrich, St. Louis, Missouri]) to culture at a density of $1.5 \times 10^{4}$ cells/well in collagen-coated 24Transwell plates (Corning, Corning, New York). We allowed organotypic cultures to proliferate with media in both apical and basal compartments starting on day in vitro (DIV) 0 . We changed the defined proliferation media every other day until DIV 7-9, when transepithelial electrical resistance exceeded $1000 \Omega \times \mathrm{cm}^{2}$, which marked the end of proliferation.

For the "Normal" phenotype, we allowed organotypic cultures to differentiate at an air-liquid interface (ALI) in a defined differentiation media (DMEM with $2 \%$ v/v NuSerum [BD BioSciences, San Jose, California], and $50 \mathrm{nM}$ retinoic acid [Sigma-Aldrich]) in the basal compartment starting on DIV 7. We changed the defined differentiation media every other day until DIV 28, which marked the end of differentiation.

For the "T2-Skewed" phenotype, we allowed organotypic cultures to differentiate at an ALI in a defined differentiation media supplemented with IL-13 (DMEM with $2 \%$ v/v NuSerum [BD BioSciences], $50 \mathrm{nM}$ retinoic acid [Sigma-Aldrich], and $25 \mathrm{ng} / \mathrm{ml}$ IL-13 [PeproTech, Rocky Hill, New Jersey]). We identified this concentration of IL-13 in a preliminary study and supplemented it to the defined proliferation media starting on DIV 5, and then to the defined differentiation media from DIV 7 to 28 , which we changed every other day until DIV 28.

AgNP exposure. We exposed organotypic cultures in the apical compartment on DIV 28 to either $2 \mathrm{mM}$ sodium citrate (vehicle control; $0 \mu \mathrm{g} \mathrm{AgNP} / \mathrm{ml}$ media), or AgNP (20 nm, gold-core, citrate-coated, at $1 \mathrm{mg} / \mathrm{ml}$ in $2 \mathrm{mM}$ sodium citrate; nanoComposix, San Diego, California) for an acute exposure of $24 \mathrm{~h}$ at nominal doses of 12.5 or $50 \mu \mathrm{g} \mathrm{AgNP} / \mathrm{ml}$ media, as freshly prepared in suspensions of defined differentiation media.

RNA-sequencing. We used RNA-seq to quantify global, differential, and targeted gene expression in exposed organotypic cultures on DIV 28. We isolated total RNA from organotypic cultures using a RNEasy Micro Kit (Qiagen, Hilden, Germany), and estimated RNA quality and quantity using an Agilent 2100 
Bioanalyzer (Agilent Technologies, Santa Clara, California) to visualize the $18 \mathrm{~S}$ and $28 \mathrm{~S}$ rRNA bands. We performed library preparation using a combination of 2 protocols which allowed for smaller volumes while minimizing handling steps, first using a SMART-seq v4 Ultra Low Input RNA Kit (Clontech Laboratories, Mountain View, California) to convert the RNA to full length cDNA and to amplify it, and then using a Nextera XT Library Preparation Kit (Fluidigm, San Francisco, California) to fragment the cDNA and add Illumina adapters. We sequenced the library products using the Illumina HiSeq2500 platform (Illumina, San Diego, California), and aligned the trimmed sequencing reads to the Ensembl mouse transcriptome (version GRCm38.84) using Spliced Transcripts Alignment to a Reference software (Dobin et al., 2013).

Quantitative reverse transcription-polymerase chain reaction. We used quantitative reverse transcription-polymerase chain reaction (qRT-PCR) to confirm gene expression for markers of T2 and T17 cytokines in exposed organotypic cultures on DIV 28. We isolated total RNA from organotypic cultures using an RNEasy Micro Kit (Qiagen, Hilden, Germany), and synthesized cDNA using a RevertAid RT Reverse Transcription Kit (ThermoFisher Scientific, Waltham, Massachusetts). We performed qRT-PCR using 20 ng cDNA per reaction with commercially available primer probe sets, and master mix (Integrated DNA Technologies, Coralville, Iowa) on a $7900 \mathrm{HT}$ Fast Real-Time PCR System (Applied BioSystems, Foster City, California). We normalized data to the $\mathrm{Ct}$ values for vehicle controls and then to the average $\mathrm{Ct}$ values of housekeeping genes Hprt1, Pol2ra, and Tbp using the $2^{-\Delta \Delta C t}$ method, as previously described in Scoville et al. (2017).

Statistics. We used average read counts $(\geq 10 ; n=9642)$ to characterize dose, genotype, and phenotype effects on: (1) global gene expression, (2) differential gene expression, (3) canonical pathway enrichment, (4) targeted gene expression for the secreted factors canonical pathway, (5) ingenuity pathway analysis (IPA) for the secreted factors canonical pathway, and (6) targeted gene expression for $\mathrm{T} 1$, pro-T2, T2, and T17 responses. We characterized hierarchical clustering of global gene expression using $z$-scores of $\log _{2}$ (average read counts) on the pheatmap package for $R$ statistical software ( $R$ Core Team, 2018). We characterized principal components of global gene expression using $\log _{2}$ (average read counts) on the DESeq2 package for $\mathrm{R}$ statistical software (R Core Team, 2018). We characterized differential gene expression using $\log _{2}$ (average read counts) with a false discovery rate $(F D R<0.10)$ to generate more robust networks and to adjust for multiple comparisons on the DESeq2 package for $\mathrm{R}$ statistical software ( $\mathrm{R}$ Core Team, 2018). We characterized canonical pathway enrichment using Gene Set Enrichment Analysis (GSEA; Broad Institute, Cambridge, Massachusetts) with the Benjamini-Hochberg method (FDR < 0.10 ) for rank-ordered $z$-scores of $\log _{2}$ (average read counts) $(n=$ 9642) on the clusterProfiler package for $R$ statistical software $(R$ Core Team, 2018). We repeated these analyses using more stringent cut-offs (FDR $<0.01$ and FDR $<0.05$ ) and observed similar numbers of differentially expressed genes (DEG) and patterns of canonical pathway enrichment. We characterized targeted gene expression for the secreted factors canonical pathway using zscores of $\log _{2}$ (average read counts) $(n=54)$ on the pheatmap package for R statistical software (R Core Team, 2018). We characterized upstream transcriptional regulators and downstream functions and diseases associated with the secreted factors canonical pathway using IPA (Ingenuity Systems, Redwood City, California).
To characterize confirmatory gene expression for T2 and T17 responses, we used linear mixed effects models to test the fixed effects of genotype, phenotype, and dose using R statistical software (R Core Team, 2018). We adjusted each model for random effects to account for variability across and within biological and technical replicates. We used ANOVA to test the significance of each fixed effect as well as interactions between these fixed effects using the nominal dose of AgNP mass as a categorical variable. We tested the significance of interactions between these fixed effects to characterize $\mathrm{G} \times \mathrm{P}$ effects on transcriptional response to AgNP toxicity. For this analysis, we considered an effect with a $p$ value less than $0.05(p<.05)$ statistically significant and adjusted all effects for multiple comparisons when comparing each genotype, phenotype, and nominal dose of AgNP mass to its vehicle control.

\section{RESULTS}

Genotype, Phenotype, and Dose Effects on Global Gene Expression We characterized dose, genotype, and phenotype effects on hierarchical clustering of global gene expression using Pearson correlation distance and average linkage distance on z-scores of $\log _{2}$ (average read counts) $(n=9642)$ for AJ:Normal (Figure 1A), B6:Normal (Figure 1B), AJ:T2-Skewed (Figure 1C), and B6:T2Skewed (Figure 1D) exposed to nominal doses of 0, 12.5, or $50 \mu \mathrm{g}$ AgNP/ml media for an acute $24 \mathrm{~h}$ exposure. We observed dose, genotype, and phenotype effects on hierarchical clustering of global gene expression, with the largest differences between the "Normal" and "T2-Skewed" phenotypes. We characterized dose, genotype, and phenotype effects on principal components of global gene expression using principal components analysis (PCA) on $\log _{2}$ (average read counts) $(n=9642)$ for AJ:Normal, B6:Normal, AJ:T2-Skewed, and B6:T2-Skewed exposed to nominal doses of 0 (Figure 2A), 12.5 (Figure 2B), or 50 (Figure 2C) $\mu \mathrm{g}$ $\mathrm{AgNP} / \mathrm{ml}$ media for an acute $24 \mathrm{~h}$ exposure. We observed dose, genotype effects on principal components of global gene expression, which explained $3 \%, 4 \%$, and $19 \%$ of the total variance at $0,12.5$, and $50 \mu \mathrm{g} \mathrm{AgNP} / \mathrm{ml}$ media, respectively. We observed phenotype effects on principal components of global gene expression, which explained $89 \%, 90 \%$, and $77 \%$ of the total variance at $0,12.5$, and $50 \mu \mathrm{g} \mathrm{AgNP} / \mathrm{ml}$ media, respectively. We observed the largest separation between AJ:T2-Skewed and B6:T2-Skewed at $50 \mu \mathrm{g}$ AgNP/ml media.

\section{Genotype, Phenotype, and Dose Effects on Differential Gene Expression}

We characterized dose, genotype, and phenotype effects on differential gene expression using a $t$ test for significant differences in $\log _{2}$ (average read counts) $(n=9642)$ for AJ:Normal, B6:Normal, AJ:T2-Skewed, and B6:T2-Skewed exposed to nominal doses of $0,12.5$, or $50 \mu \mathrm{g}$ AgNP/ml media for an acute $24 \mathrm{~h}$ exposure (FDR $<0.10$ ). We observed dose effects on upregulated DEG, with 49 and 38 genes uniquely expressed in AJ:Normal (12.5 and $50 \mu \mathrm{g} \mathrm{AgNP/ml} \mathrm{media} \mathrm{vs} \mathrm{vehicle} \mathrm{controls,} \mathrm{respectively),}$ 2 and 4 genes uniquely expressed in B6:Normal (12.5 and $50 \mu \mathrm{g}$ AgNP/ml media vs vehicle controls, respectively), 15 and 122 genes uniquely expressed in AJ:T2-Skewed (12.5 and $50 \mu \mathrm{g}$ $\mathrm{AgNP} / \mathrm{ml}$ media vs vehicle controls, respectively), and 188 and 163 genes uniquely expressed in B6:T2-Skewed (12.5 and $50 \mu \mathrm{g}$ $\mathrm{AgNP} / \mathrm{ml}$ media vs vehicle controls, respectively) (Supplementary Figure 1). We observed dose effects on downregulated DEG, with 43 and 262 genes uniquely expressed in AJ:Normal (12.5 and $50 \mu \mathrm{g}$ AgNP/ml media vs vehicle controls, 


\section{GxP Effects on Hierarchical Clustering of Global Gene Expression}

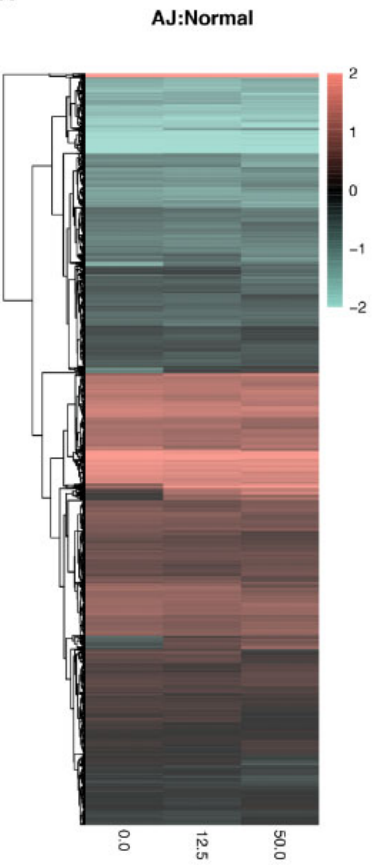

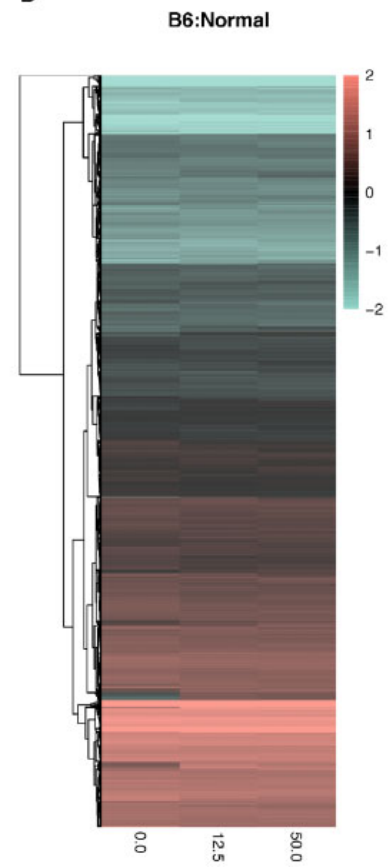

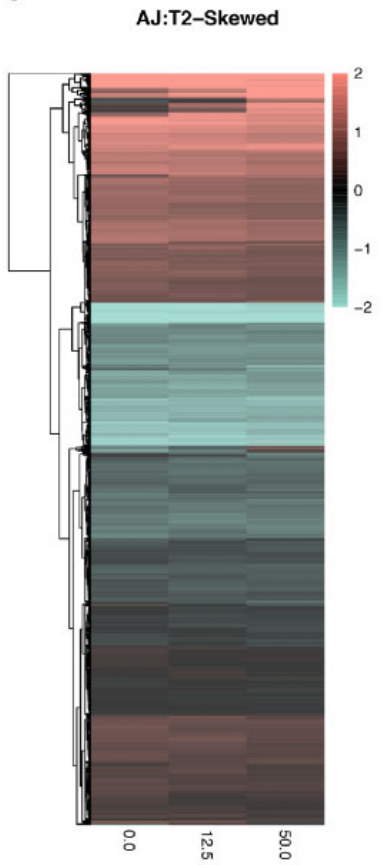

B6:T2-Skewed

Figure 1. Genotype, phenotype, and dose effects on hierarchical clustering of global gene expression. Hierarchical clustering of global gene expression was characterized using Pearson correlation distance and average linkage distance of $\log _{2}$ (average read counts) ( $\left.n=9642\right)$ for AJ:Normal (A), B6:Normal (B), AJ:T2-Skewed (C), and B6:T2-Skewed (D) exposed to nominal doses of 0, 12.5, or $50 \mu \mathrm{g}$ AgNP/ml media for an acute $24 \mathrm{~h}$ exposure. Data represent average $z$-scores (red, positive; green, negative); rows represent genes, and columns represent genotypes and phenotypes. $n=3$ biological replicates.

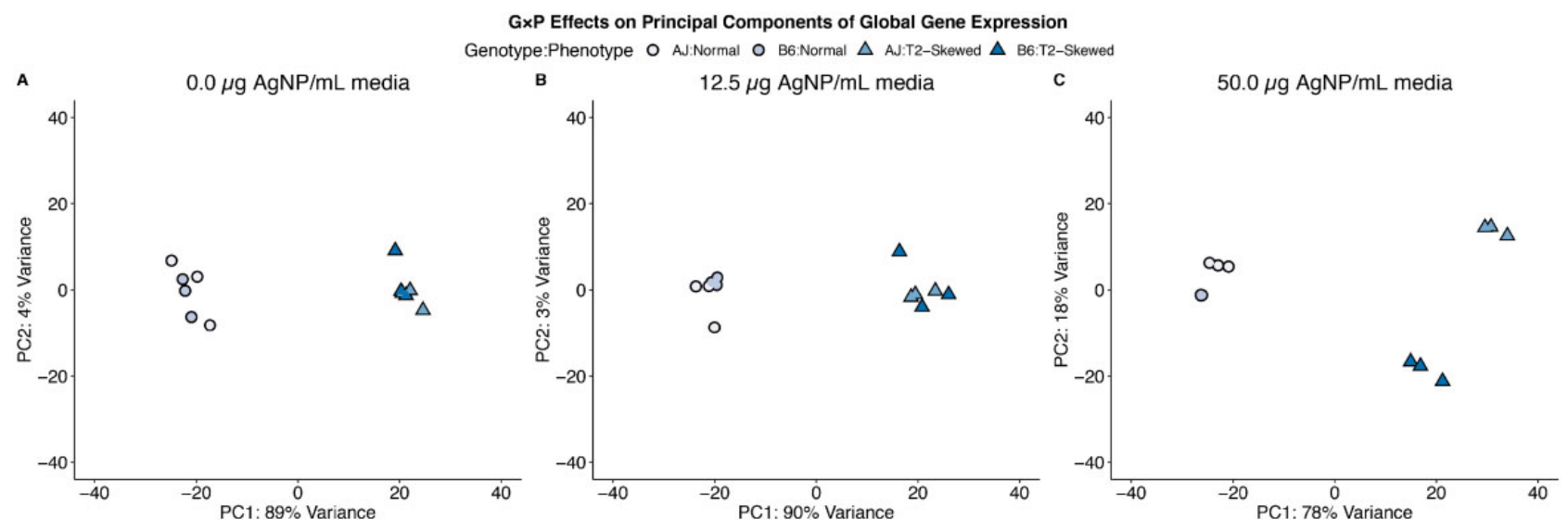

Figure 2. Genotype, phenotype, and dose effects on principal components of global gene expression. Principal components of global gene expression were characterized using PCA of $\log _{2}$ (average read counts) $(n=9642)$ for AJ:Normal, B6:Normal, AJ:T2-Skewed, and B6:T2-Skewed exposed to nominal doses of 0 (A), 12.5 (B), or 50 (C) $\mu \mathrm{g} \mathrm{AgNP} / \mathrm{ml}$ media for an acute $24 \mathrm{~h}$ exposure. $n=3$ biological replicates.

respectively), 4 and 37 genes uniquely expressed in B6:Normal (12.5 and $50 \mu \mathrm{g} \mathrm{AgNP/ml} \mathrm{media} \mathrm{vs} \mathrm{vehicle} \mathrm{controls,} \mathrm{respectively),}$ 84 and 93 genes uniquely expressed in AJ:T2-Skewed (12.5 and $50 \mu \mathrm{g} \mathrm{AgNP/ml} \mathrm{media} \mathrm{vs} \mathrm{vehicle} \mathrm{controls,} \mathrm{respectively),} \mathrm{and} 160$ and 202 genes uniquely expressed in B6:T2-Skewed (12.5 and 50 $\mu \mathrm{g} \mathrm{AgNP} / \mathrm{ml}$ media vs vehicle controls, respectively) (Supplementary Figure 1).

\section{Genotype, Phenotype, and Dose Effects on Canonical Pathway} Enrichment

We characterized dose, genotype, and phenotype effects on canonical pathway enrichment using GSEA with the
Benjamini-Hochberg method (FDR $<0.10$ ) on rank-ordered $z$-scores of $\log _{2}$ (average read counts) $(n=9642)$ for AJ:Normal, B6:Normal, AJ:T2-Skewed, and B6:T2-Skewed exposed to nominal doses of $0,12.5$, or $50 \mu \mathrm{g}$ AgNP/ml media for an acute $24 \mathrm{~h}$ exposure (Figure 3, Supplementary Table 1). We defined directionality of enrichment using a normalized enrichment score $>0$ to represent activation, and a normalized enrichment score $<0$ to represent inhibition.

The top activated canonical pathway predicted for AJ:Normal at $0 \mu \mathrm{g}$ AgNP/ml media was "N-Glycan Trimming" $(n=12$ genes; $\log [p$ value $]=2.70)$, and the top inhibited canonical pathway predicted for AJ:Normal at $0 \mu \mathrm{g}$ AgNP/ml media was 

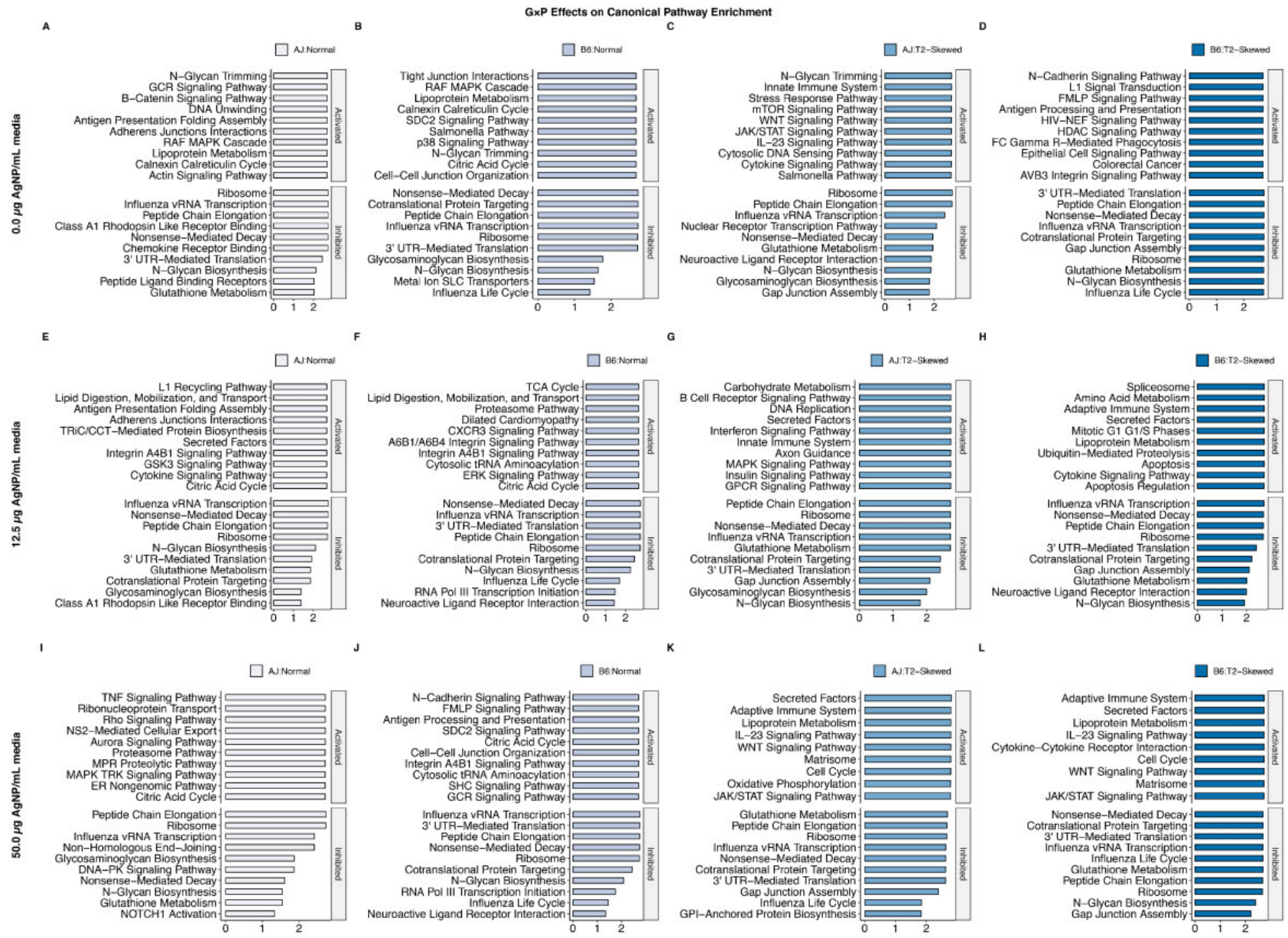

Figure 3. Genotype, phenotype, and dose effects on canonical pathway enrichment. Canonical pathway enrichment was characterized using Gene Set Enrichment Analysis with the Benjamini-Hochberg method (FDR < 0.10) on rank-ordered z-scores of $\log _{2}$ (average read counts) ( $\left.n=9642\right)$ for AJ:Normal, B6:Normal, AJ:T2-Skewed, and B6:T2-Skewed exposed to nominal doses of 0 (A-D), 12.5 (E-H), or 50 (I, J) $\mu$ g AgNP/ml media for an acute 24 h exposure. Data represent the top 10 enriched canonical pathways with significant $\log (p$ values), where activated, normalized enrichment score $>0$; inhibited, normalized enrichment score $<0$. $n=3$ biological replicates.

"Ribosome" ( $n=50$ genes; $\log [p$ value $]=2.74$ ) (Figure $3 \mathrm{~A})$. The top activated canonical pathway predicted for AJ:Normal at 12.5 $\mu \mathrm{g}$ AgNP/ml media was "L1 Recycling Pathway" ( $n=20$ genes; $\log [p$ value $]=2.68)$, and the top inhibited canonical pathway predicted for AJ:Normal at $12.5 \mu \mathrm{g}$ AgNP/ml media was "Influenza vRNA Transcription" ( $n=64$ genes; $\log [p$ value $]=$ 2.76) (Figure 3E). The top activated canonical pathway predicted for AJ:Normal at $50 \mu \mathrm{g}$ AgNP/ml media was "TNF Signaling Pathway" ( $n=27$ genes; $\log [p$ value $]=2.71$ ), and the top inhibited canonical pathway predicted for AJ:Normal at $50 \mu \mathrm{g}$ AgNP/ $\mathrm{ml}$ media was "Peptide Chain Elongation" ( $n=49$ genes; $\log [p$ value $=2.73$ (Figure 3I).

The top activated canonical pathway predicted for B6:Normal at $0 \mu \mathrm{g}$ AgNP/ml media was "Tight Junction Interactions" ( $n=10$ genes; $\log [p$ value $]=2.68$ ), and the top inhibited canonical pathway predicted for B6:Normal at $0 \mu \mathrm{g}$ AgNP/ml media was "Nonsense-Mediated Decay" ( $n=70$ genes; $\log [p$ value $]=2.73$ ) (Figure $3 B$ ). The top activated canonical pathway predicted for B6:Normal at $12.5 \mu \mathrm{g} \mathrm{AgNP} / \mathrm{ml}$ media was "TCA Cycle" ( $n=18$ genes; $\log [p$ value $]=2.66)$, and the top inhibited canonical pathway predicted for B6:Normal at $12.5 \mu \mathrm{g}$ AgNP/ml media was "Nonsense-Mediated Decay" ( $n=70$ genes; $\log [p$ value $]=2.75$ ) (Figure $3 F$ ). The top activated canonical pathway predicted for B6:Normal at $50 \mu \mathrm{g}$ AgNP/ml media was "N-Cadherin Signaling Pathway" ( $n=26$ genes; $\log [p$ value $]=$ 2.70), and the top inhibited canonical pathway predicted for B6:Normal at $50 \mu \mathrm{g} \mathrm{AgNP/ml} \mathrm{media} \mathrm{was} \mathrm{"Influenza} \mathrm{vRNA}$ Transcription" ( $n=64$ genes; $\log [p$ value $]=2.75$ ) (Figure 3J).

The top activated canonical pathway predicted for AJ:T2Skewed at $0 \mu \mathrm{g}$ AgNP/ml media was "N-Glycan Trimming" ( $n=$ 12 genes; $\log [p$ value $]=2.70$ ), and the top inhibited canonical pathway predicted for AJ:T2-Skewed at $0 \mu \mathrm{g}$ AgNP/ml media was "Ribosome" ( $n=50$ genes; $\log [p$ value $]=2.73$ ) (Figure $3 C$ ). The top activated canonical pathway predicted for AJ:T2-Skewed at $12.5 \mu \mathrm{g}$ AgNP/ml media was "Carbohydrate Metabolism" $(n=141$ genes; $\log [p$ value $]=2.72$ ), and the top inhibited canonical pathway predicted for AJ:T2-Skewed at $12.5 \mu \mathrm{g} \mathrm{AgNP} / \mathrm{ml}$ media was "Peptide Chain Elongation" ( $n=49$ genes; $\log [p$ value $]=2.71$ ) (Figure 3G). The top activated canonical pathway predicted for AJ:T2-Skewed at $50 \mu \mathrm{g} \mathrm{AgNP/ml} \mathrm{media} \mathrm{was} \mathrm{"Secreted} \mathrm{Factors"} \mathrm{(} n$ $=98$ genes; $\log [p$ value $]=2.80)$, and the top inhibited canonical pathway predicted for AJ:T2-Skewed at $50 \mu \mathrm{g} \mathrm{AgNP/ml} \mathrm{media}$ was "Glutathione Metabolism" ( $n=34$ genes; $\log [p$ value $]=2.67$ ) (Figure 3K).

The top activated canonical pathway predicted for B6:T2Skewed at $0 \mu \mathrm{g}$ AgNP/ml media was "N-Cadherin Signaling 
Pathway" ( $n=26$ genes; $\log [p$ value $]=2.70)$, and the top inhibited canonical pathway predicted for B6:T2-Skewed at $0 \mu \mathrm{g}$ AgNP/ml media was " 3 ' UTR-Mediated Translation" $(n=67$ genes; $\log [p$ value $]=2.70$ ) (Figure 3D). The top activated canonical pathway predicted for B6:T2-Skewed at $12.5 \mu \mathrm{g}$ AgNP/ml media was "Spliceosome" ( $n=118$ genes; $\log [p$ value $]=2.72$ ), and the top inhibited canonical pathway predicted for B6:T2-Skewed at $12.5 \mu \mathrm{g} \mathrm{AgNP/ml} \mathrm{media} \mathrm{was} \mathrm{"Influenza} \mathrm{vRNA} \mathrm{Transcription"}$ $(n=64$ genes; $\log [p$ value $]=2.70$ ) (Figure $3 \mathrm{H})$. The top activated canonical pathway predicted for B6:T2-Skewed at $50 \mu \mathrm{g} \mathrm{AgNP/}$ $\mathrm{ml}$ media was "Adaptive Immune System" $(n=349$ genes; $\log [p$ value] $=2.74$ ), and the top inhibited canonical pathway predicted for B6:T2-Skewed at $50 \mu \mathrm{g} \mathrm{AgNP} / \mathrm{ml}$ media was "Nonsense-Mediated Decay" ( $n=70$ genes; $\log [p$ value $]=2.70$ ) (Figure 3L).

\section{Genotype, Phenotype, and Dose Effects on Targeted Gene Expression for the Secreted Factors Canonical Pathway}

We identified "Secreted Factors" as an important enriched canonical pathway that may play a role in defining the mechanistic basis for $\mathrm{G} \times \mathrm{P}$ effects on AgNP toxicity. To better understand this mechanistic basis, we characterized dose, genotype, and phenotype effects on targeted gene expression for the secreted factors canonical pathway using $\mathrm{z}$-scores of $\log _{2}$ (average read counts) $(n=54)$ for AJ:Normal (Figure 4A), B6:Normal (Figure 4B), AJ:T2-Skewed (Figure 4C), and B6:T2-Skewed (Figure 4D) exposed to nominal doses of $0,12.5$, or $50 \mu \mathrm{g}$ AgNP/ml media for an acute $24 \mathrm{~h}$ exposure. We chose this subset of genes based on leading-edge analysis, which identifies the subsets of genes (referred to as the leading-edge subset) that contributed the most to the enrichment score of a given gene set's core enrichment. We observed dose, genotype, and phenotype effects on targeted gene expression, specifically upregulated genes for chemokines responsible for attracting leukocyte populations including $\mathrm{T}$ lymphocytes (eg, Ccl20 and Ccl22), dendritic cells (eg, Ccl25), eosinophils (eg, Ccl24 and Ccl26), and neutrophils (eg, Cxcl1, Cxcl2, Cxcl3, and Cxcl5). We observed dose, phenotype effects on upregulated genes for cytokines associated with T2/T17 responses, specifically upregulated genes for T2 cytokines in the "Normal" phenotype (eg, Il4, Il5, Il6, Il9, Il10, and Il13), and upregulated genes for T17 cytokines in the "T2-Skewed" phenotype (eg, Ccl20, Ccl25, Csf3, Cxcl1, Cxcl2, Cxcl3, Cxcl5, Il17c, and Il23a).

Genotype, Phenotype, and Dose Effects on Upstream Transcriptional Regulation of the Secreted Factors Canonical Pathway

To further understand this mechanistic basis, we also characterized dose, genotype, and phenotype effects on upstream regulators, downstream functions, and diseases of the secreted factors canonical pathway using IPA with the Fischer's Exact method (FDR $<0.10$ ) on fold changes of $\log _{2}$ (average AgNP/vehicle control) $(n=54)$ for AJ:Normal (Figs 5A and 6A), B6:Normal (Figs $5 B$ and $6 A$ ), AJ:T2-Skewed (Figs $5 C$ and $6 C$ ), and B6:T2Skewed (Figs 5D and 6D) exposed to nominal doses of $0,12.5$, or $50 \mu \mathrm{g} \mathrm{AgNP} / \mathrm{ml}$ media for an acute $24 \mathrm{~h}$ exposure. These predictions were based on the overlap $p$ value, which measures the significance of the overlap between affected genes and all of the genes within the secreted factors canonical pathway. We used the upstream analysis module of IPA to identify dose, genotype, and phenotype effects on upstream regulators of the secreted factors canonical pathway. Across doses, genotypes, and phenotypes, we identified Stat3 as one of the most significant upstream activators of the secreted factors canonical pathway (Figure 5), and Bcl6 as one of the most significant upstream inhibitors of the secreted factors canonical pathway (Figure 6).
We used the pathway analysis module of IPA to identify dose, genotype, and phenotype effects on specific cytokines that may serve as upstream activators of the T17 canonical pathway. Across doses, genotypes, and phenotypes, we identified IL-23 as the most significant upstream activator of STAT3 within the T17 canonical pathway (Supplementary Figs 5-12). We used the downstream analysis module of IPA to identify dose, genotype, and phenotype effects on downstream functions and diseases of the secreted factors canonical pathway. Across doses, genotypes, and phenotypes, we identified neutrophil chemotaxis as one of the most significant downstream functions of the secreted factors canonical pathway (Supplementary Figure 13), and lung inflammation as one of the most significant downstream diseases of the secreted factors canonical pathway (Supplementary Figure 14).

\section{Genotype, Phenotype, and Dose Effects on Targeted Gene Expression} for T1, pro-T2, T2, and T17 Responses

We identified dose, genotype, and phenotype effects on IL-23mediated STAT3 activation, which may define a potential mechanistic basis for $\mathrm{G} \times \mathrm{P}$ effects on AgNP toxicity. To better understand how this mechanism may differentially regulate AgNP-induced T1/pro-T2/T2/T17 responses, we characterized dose effects on targeted gene expression for T1, pro-T2, T2, and $\mathrm{T} 17$ cytokines. We observed discordant regulation across $\mathrm{T} 1$, pro-T2, T2, and T17 responses. We observed no consistent dose, genotype, or phenotype effects on AgNP-induced T1 responses (Figs. 7A and 7B). However, we observed dose, phenotype effects on AgNP-induced pro-T2 (Figs. 7C and 7D) and T2 responses (Figs. 7E and 7F), with downregulation of upstream pro-T2 cytokines Il25, Il33, and Tslp as well as downstream T2 cytokines Il4, Il5, Il6, Il9, Il10, and Il13 under the "T2-Skewed" phenotype $(p<$ $.05-\mathrm{NS})$ compared with the "Normal" phenotype $(p<0.001)$. We observed dose, phenotype effects on AgNP-induced regulation of T17 responses (Figs. 7G and 7H), with upregulation of T17 activator Stat3 and downregulation of T17 inhibitor Bcl6 under the "T2-Skewed" phenotype $(p<.001-p>.05)$ compared with the "Normal" phenotype $(p<.001-p>.05)$. We observed dose, phenotype effects on AgNP-induced T17 responses (Figs. 7G and $7 \mathrm{H})$, with stronger, more persistent upregulation of T17 cytokines Ccl20, Ccl25, Csf3, Cxcl1, Cxcl2, Cxcl3, Cxcl5, Il17c, Il23a, and Tgfb1 under the "T2-Skewed" phenotype $(p<.001)$ compared with the "Normal" phenotype $(p<.001-p>.05)$. The observed dose, phenotype effects on targeted gene expression suggest $\mathrm{G} \times \mathrm{P}$ effects on the "T2-Skewed" phenotype's shift toward a "T17-Like" phenotype, or an in vitro model of severe chronic respiratory diseases characteristic of neutrophilic/difficult-tocontrol asthma.

\section{Genotype, Phenotype, and Dose Effects on Confirmatory Gene Expression for T2 and T17 Responses}

We did not observe genotype effects on confirmatory gene expression $(p>.05)$; however, we observed phenotype effects on confirmatory gene expression $(p<.001)$ (Figure 8). We observed confirmatory gene expression under the "T2-Skewed" phenotype recapitulated clinical features of severe chronic respiratory diseases through a shift toward T17 responses. We observed reciprocal regulation of T2/T17 responses under the "T2-Skewed" phenotype by downregulation of T2 cytokines Il6, Il10, and Il13 (Figs. 8A and 8B) and upregulation of T17 cytokines Cxcl1, Cxcl2, and Cxcl5 (Figs. 8C and 8D). We observed dose effects on confirmatory expression for Cxcl1 and Cxcl5 under the "Normal" phenotype $(p<.01)$ as well as Cxcl1, Cxcl2, and Cxcl5 under the "T2-Skewed" phenotype $(p<.001)$. In addition, we observed 
GxP Effects on the Secreted Factors Canonical Pathway

A

\section{AJ:Normal}

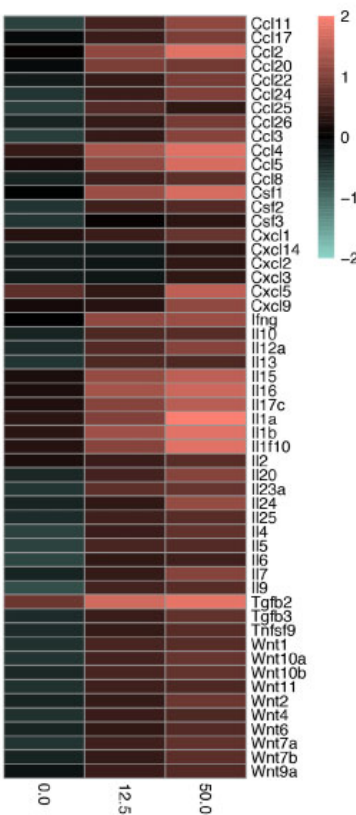

B6:Normal

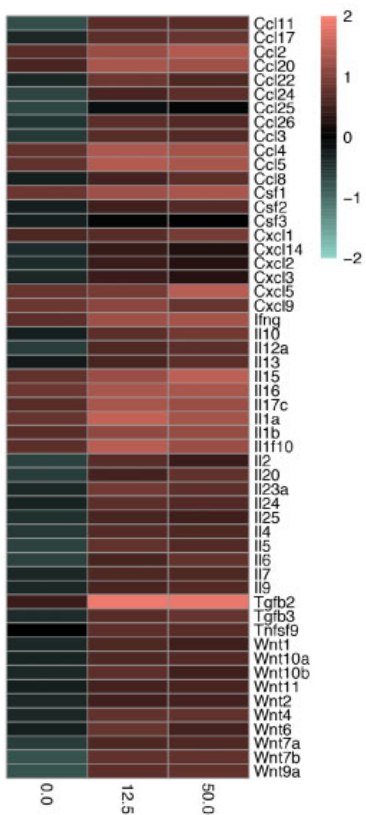

AJ:T2-Skewed

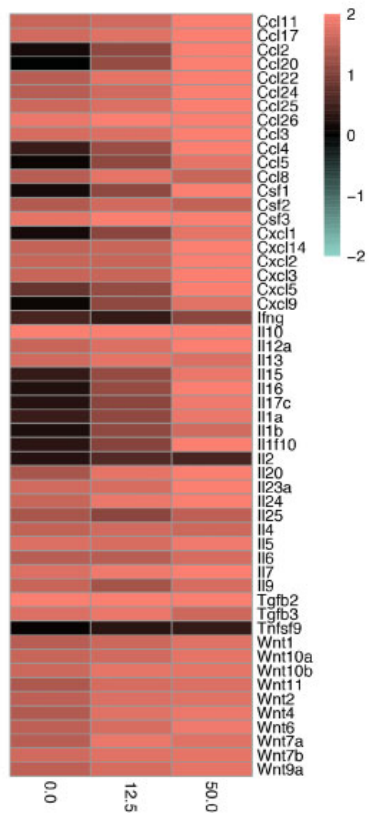

B6:T2-Skewed

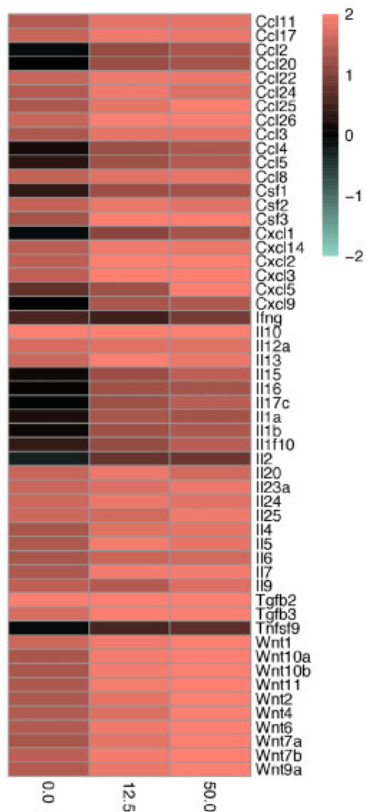

Figure 4. Genotype, phenotype, and dose effects on targeted gene expression for the secreted factors canonical pathway. Targeted gene expression for the secreted factors canonical pathway was characterized using z-scores of $\log _{2}$ (average read counts) ( $\left.n=54\right)$ for AJ:Normal (A), B6:Normal (B), AJ:T2-Skewed (C), and B6:T2-Skewed (D) exposed to nominal doses of $0,12.5$, or $50 \mu \mathrm{g} \mathrm{AgNP/ml} \mathrm{media} \mathrm{for} \mathrm{an} \mathrm{acute} 24 \mathrm{~h}$ exposure. Data represent average $\mathrm{z}$-scores (red, positive; green, negative); rows represent genes, and columns represent doses. $n=3$ biological replicates.

$\mathrm{G} \times \mathrm{P}$ effects on confirmatory gene expression for each genes and dose at the acute $24 \mathrm{~h}$ exposure $(p<.001)$.

\section{DISCUSSION}

This is the first study to use organotypic cultures as a highcontent in vitro model of the conducting airway to characterize $\mathrm{G} \times \mathrm{P}$ effects on transcriptional response to AgNP toxicity. We characterized global, differential, and targeted gene expression, canonical pathway enrichment, and upstream transcriptional regulation to understand $G \times P$ effects on transcriptional response to AgNP toxicity. We observed the "T2-Skewed" phenotype was marked by increased pro-inflammatory T17 responses to AgNP toxicity, which are significant predictors of neutrophilic/difficult-to-control asthma. This suggests that asthmatics could be a sensitive population to AgNP exposures in occupational settings, as these adverse cellular responses may further modulate innate and adaptive immune responses at the epithelial-immune interface to exacerbate this chronic airway disease (Alsaleh and Brown, 2018). Understanding $G \times P$ effects is important for identifying these sensitive populations, whose underlying genetics or diseases could directly modify their response to AgNP exposures.

Asthma is a heterogeneous chronic airway disease regulated by divergent mechanisms of $\mathrm{T} 2$ and $\mathrm{T} 17$ inflammation. In a cross-sectional study by Brown et al., observed IL-4 and IL-13 were significant predictors of the T2-high endotype, or eosinophilic/easy-to-control asthma, whereas CXCL1, IL-5, IL-8, and IL-17A were significant predictors of the T17-high endotype, or neutrophilic/difficult-to-control asthma (Brown et al., 2017). In another cross-sectional study by Choy et al., endobronchial tissue gene expression in asthmatics revealed 3 distinct endotypes of increasing severity: T2/17-low, T2-high, and T17-high (Choy et al., 2015). To better understand this heterogeneity, the authors investigated how suppression of $\mathrm{T} 2$ cytokines may drive the potentiation of T17 responses in house dust mite (HDM)-sensitized, female BALB/CJ mice injected with anti-IL-4, anti-IL-13, and anti-IL-17 antibodies prior to sensitization. They observed that neutralization of IL-4 and/or IL-13 produced increased levels of T17 cells and exacerbated T17 lung inflammation, and that neutralization of both IL-13 and IL-17A was protective against mucus production, airway remodeling, and hyperresponsiveness, as well as T2/T17 lung inflammation, suggesting that combination therapies targeting both mechanisms may maximize therapeutic efficacy in asthmatics comprising both T2-high and T17-high endotypes. These cytokines are differentially regulated across endotypes and have the potential to be shifted by AgNP toxicity. In this study, we observed dose, phenotype effects on AgNP-induced T17 responses, with stronger, more persistent upregulation of T17 cytokines Ccl20, Ccl25, Csf3, Cxcl1, Cxcl2, Cxcl3, Cxcl5, Il17c, Il23a, and Tgfb1 under the "T2-Skewed" phenotype compared with the "Normal" phenotype. The observed dose, phenotype effects on targeted gene expression suggest these cytokines are differentially regulated across phenotypes and were shifted by AgNP toxicity.

Our observations are supported by previous in vivo studies on the effects of host genetic factors on AgNP toxicity. Multiple in vivo studies treated ovalbumin (OVA)-sensitized, female $\mathrm{BALB} / \mathrm{CJ}$ or C57BL6/J mice with AgNP of various sizes and coatings. Although some of these studies have suggested attenuation of their allergic airway phenotype (Park et al., 2010), others have found exacerbation of their allergic airway phenotype through increased AgNP-induced mucus production (marked by 


\section{G×P Effects on STAT3 Activation Upstream of the Secreted Factors Canonical Pathway}

A 12.5 vs. $0.0 \mu \mathrm{g} \mathrm{AgNP} / \mathrm{mL}$ media AJ:Normal

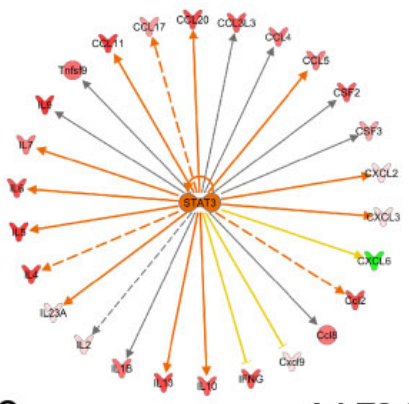
50.0 vs. $0.0 \mu \mathrm{g} \mathrm{AgNP} / \mathrm{mL}$ media

AJ:T2-Skewed 12.5 vs. $0.0 \mu \mathrm{g} \mathrm{AgNP} / \mathrm{mL}$ media

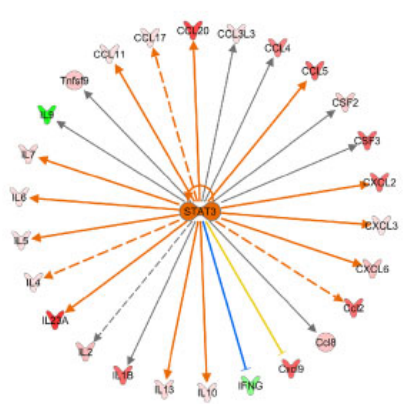

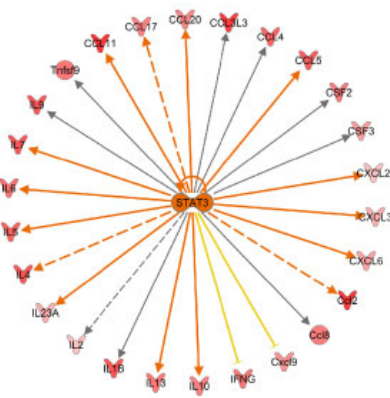

50.0 vs. $0.0 \mu \mathrm{g} \mathrm{AgNP} / \mathrm{mL}$ media
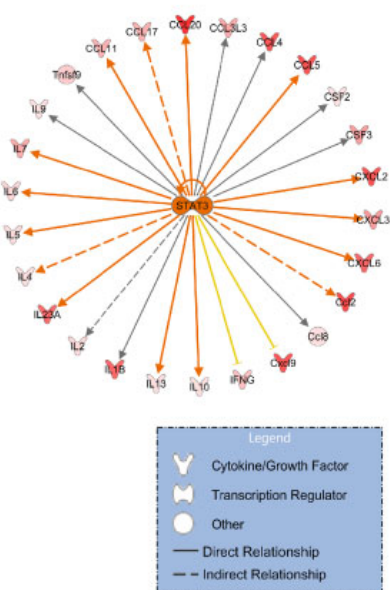

B 12.5 vs. $0.0 \mu \mathrm{g} \mathrm{AgNP} / \mathrm{mL}$ media B6:Normal

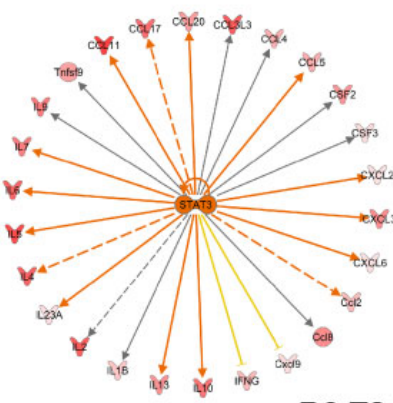

B6:T2-Skewed 12.5 vs. $0.0 \mu \mathrm{g} \mathrm{AgNP} / \mathrm{mL}$ media

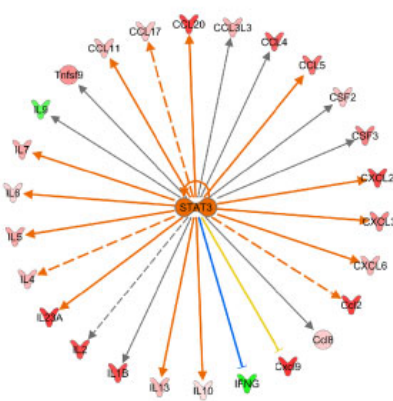

Upregulation

Downregulation

Predicted activation
Predicted inhibition

Predicted inhibition

Unpredicted relationship 50.0 vs. $0.0 \mu \mathrm{g} \mathrm{AgNP} / \mathrm{mL}$ media

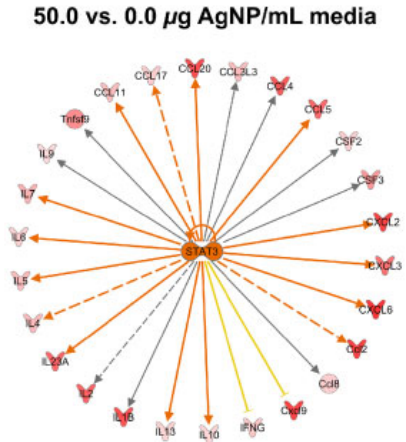

50.0 vs. $0.0 \mu \mathrm{g} \mathrm{AgNP} / \mathrm{mL}$ media

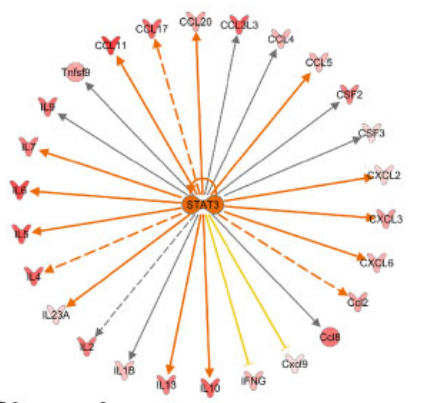

Figure 5. Genotype, phenotype, and dose effects on Stat3 activation upstream of the secreted factors canonical pathway. Upstream activation of the secreted factors canonical pathway was characterized using IPA with the Fischer's Exact method (FDR $<0.10$ ) on fold changes of $\log _{2}$ (average AgNP/vehicle control) $(n=54)$ for AJ:Normal (A), B6:Normal (B), AJ:T2-Skewed (C), and B6:T2-Skewed (D) exposed to nominal doses of 0, 12.5, or $50 \mu \mathrm{g}$ AgNP/ml media for an acute $24 \mathrm{~h}$ exposure. Central icons represent the upstream activator (Stat3), peripheral icons represent cytokine/growth factors, and arrows represent predicted relationships. $n=3$ biological replicates.

increased phosphatidylinositide 3-kinase/hypoxia-inducible factor 1 alpha/vascular endothelial growth factor signaling pathway activity, and decreased mucin 5AC signaling pathway activity), oxidative stress (marked by increased ROS production, $\mathrm{NF}-\kappa \mathrm{B}$ activation, leukotriene $\mathrm{E} 4$ and 8-hydroxy-2'-deoxyguanosine production), and T2 lung inflammation (marked by increased T1/2 cytokine secretion, pro-inflammatory cell infiltrate, immunoglobulin $\mathrm{E}$, and AHR) in allergic mice compared with healthy mice and filtered air controls (Chuang et al., 2013; Jang et al., 2012; Su et al., 2013). These previous in vivo studies used the OVA combined with exogenous alum model, which is T-cell dependent. Their findings may be less relevant in a clinically relevant model of asthma, which uses a complete allergen such as HDM and is more dependent on innate immune and airway epithelial cell responses (Hallstrand et al., 2014; Hammad et al., 2009). Thus, these disparate findings in previous in vivo studies using the OVA/alum model do not negate the potential impact of phenotype effects on transcriptional response to AgNP toxicity.

Several in vivo studies have also characterized a similar exacerbation of this allergic airway phenotype in male BrownNorway rats. Seiffert et al. exposed male Brown-Norway and Sprague-Dawley rats, and observed size, coating, and genotype effects on AgNP-induced T2/T17 lung inflammation (marked by increased IL-6 and IL-17A secretion, pro-inflammatory cell infiltrate, and changes in histopathology) and lung dysfunction (marked by decreased airway resistance, tissue elastance), with Brown-Norway rats being the more sensitive genotype (Seiffert et al., 2015, 2016). The authors also quantified dosimetric doses of silver (Ag) mass in the lungs of these differentially sensitive genotypes and detected higher burdens in Brown-Norway rats compared with Sprague-Dawley rats, suggesting that airway physiology (marked by alveolar size and airway branching) may be a contributing factor in genotype effects on AgNP toxicity, marked by AgNP-induced T2/T17 lung inflammation. Our observations were also supported by IPA in this study, which identified Stat3 as one of the most significantly activated upstream transcriptional regulators, and Bcl6 as one of the most significantly inhibited upstream transcriptional regulators of the secreted factors canonical pathway. Stat3 is recognized as the essential regulator of T17 cells, analogous to Stat1 and Stat4 in T1 cells, and Stat6 in T2 cells. Il6 and Il23a have been shown to synergistically regulate Stat3 in airway epithelial cells (Simeone-Penney et al., 2007), and whereas IL-23 is mostly produced by dendritic cells and macrophages, Lee et al. observed that this IL-17 inducible cytokine was produced in airway epithelial cells derived from antigen-sensitized female BALB/cJ mice (Lee et al., 2017). IL-23 induces the differentiation of naive $\mathrm{CD}^{+}$T-cells into T17 cells; inhibiting IL-23 or its downstream factors, including IL-17, can attenuate the progression of 


\section{G×P Effects on BCL6 Inhibition Upstream of the Secreted Factors Canonical Pathway}

A 12.5 vs. $0.0 \mu \mathrm{g} \mathrm{AgNP} / \mathrm{mL}$ media AJ:Normal

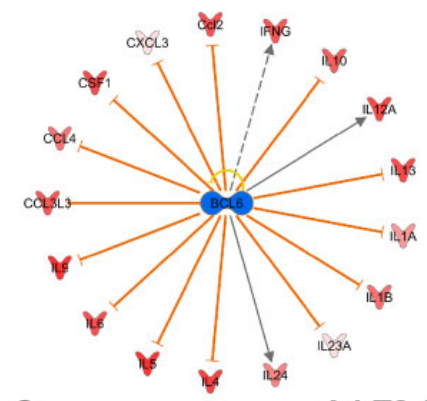
50.0 vs. $0.0 \mu \mathrm{g} \mathrm{AgNP/mL} \mathrm{media}$

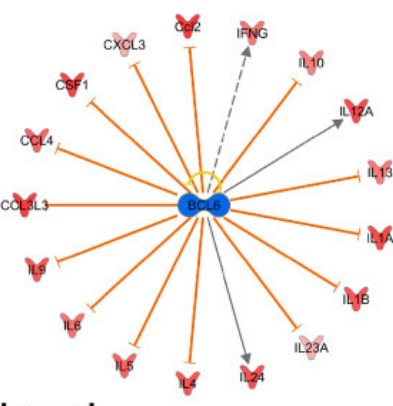

AJ:T2-Skewed

12.5 vs. $0.0 \mu \mathrm{g} \mathrm{AgNP} / \mathrm{mL}$ media

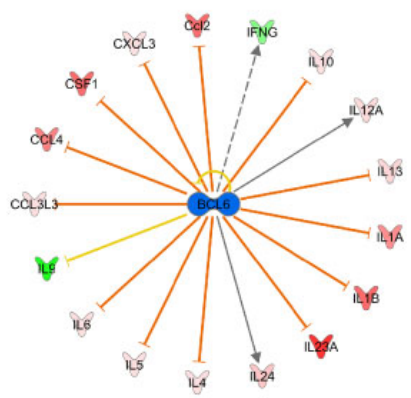

50.0 vs. $0.0 \mu \mathrm{g} \mathrm{AgNP} / \mathrm{mL}$ media

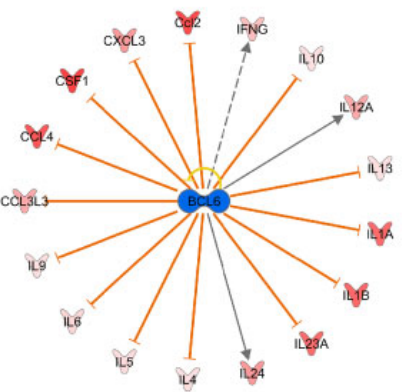

B 12.5 vs. $0.0 \mu \mathrm{g} \mathrm{AgNP/mL}$ media
B6:Normal

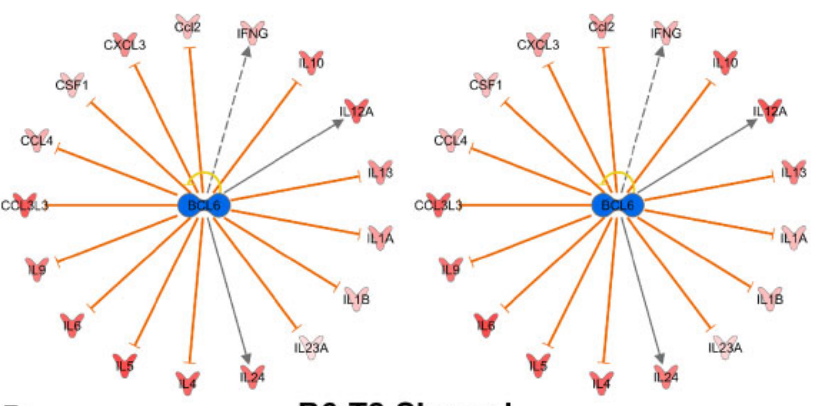

B6:T2-Skewed
50.0 vs. $0.0 \mu \mathrm{g} \mathrm{AgNP} / \mathrm{mL}$ media
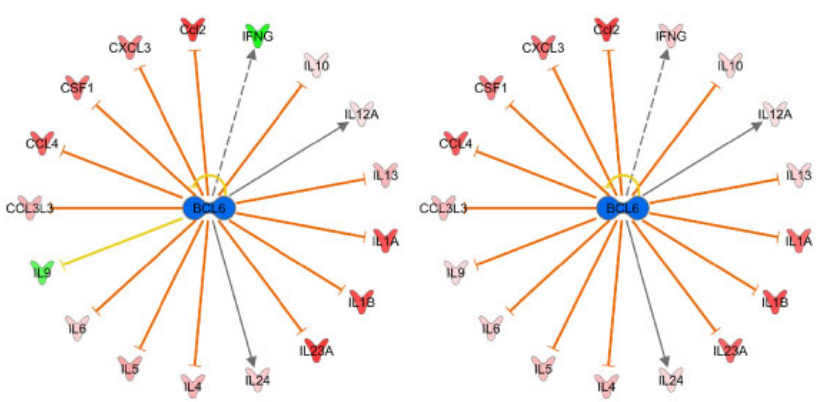

Upregulation

Downregulation

Predicted activation

Predicted inhibition

Unpredicted relationship

Figure 6. Genotype, phenotype, and dose effects on Bcl6 inhibition upstream of the secreted factors canonical pathway. Upstream inhibition of the secreted factors canonical pathway was characterized using IPA with the Fischer's Exact method (FDR $<0.10$ ) on fold changes of $\log _{2}$ (average AgNP/vehicle control) $(n=54)$ for AJ:Normal (A), B6:Normal (B), AJ:T2-Skewed (C), and B6:T2-Skewed (D) exposed to nominal doses of 0, 12.5, or $50 \mu \mathrm{g}$ AgNP/ml media for an acute $24 \mathrm{~h}$ exposure. Central icons represent the upstream inhibitor (Bcl6), peripheral icons represent cytokine/growth factors, and arrows represent predicted relationships. $n=3$ biological replicates.

inflammatory bowel diseases in animal models, which suggests the IL-23/IL-17 axis's relevance as a therapeutic target for chronic respiratory diseases (Yen et al., 2006). Stat3 has been shown to regulate Bcl6 (Wu et al., 2015), which is recognized as another essential regulator of T17 cells. Mondal et al. observed that Bcl6 deficiency promoted T17 responses through suppression of T2 responses in Bcl6-knockout, C57BL/6J-129Sv mice (Mondal et al., 2010). In this study, the shift toward a "T17Like" phenotype may have also been orchestrated by reciprocal regulation of Stat3 and Bcl6 to suppress T2 cytokines Il4, Il5, Il6, Il9, Il10, and Il13. In addition to Stat3 and Bcl6, T17 responses have also been shown to be tightly regulated by TGF- $\beta$ and Wnt signaling (Lee et al., 2014; Suryawanshi et al., 2015). However, in this study, we observed more limited dose, genotype, and phenotype effects on TGF- $\beta$ and Wnt signaling canonical pathway enrichment, which suggests these canonical pathways may have played a more understated role when compared with the secreted factors canonical pathway with regard to driving the mechanistic basis for $G \times P$ effects on AgNP toxicity. Overall, the observed dose, genotype, and phenotype effects on IL-23-mediated Stat3 activation are suggestive of $\mathrm{G} \times \mathrm{P}$ effects on upstream transcriptional regulation of the secreted factors canonical pathway, which produced a shift toward a "T17-Like" phenotype, or an in vitro model of severe chronic respiratory diseases characteristic of neutrophilic/difficult-to-control asthma.

This is first study to use organotypic cultures as a highcontent in vitro model of the conducting airway paired with RNA-seq to characterize $\mathrm{G} \times \mathrm{P}$ effects on transcriptional response to AgNP toxicity, and therefore, refining this in vitro model in future studies is warranted. One limitation of this study was the use of only 1 sex and 2 genotypes; the use of both sexes and additional genotypes (eg, BALB/cJ and SWR/J mice) or knockouts (eg, Il $23^{-/-}$, Stat $3^{-/-}$, or Bcl6 $6^{-/-}$mice) would increase genetic diversity and further assist in defining a mechanistic basis for $\mathrm{G} \times \mathrm{P}$ effects on AgNP toxicity in future studies. A second limitation of this study was the use of IL-13 to skew differentiation toward an in vitro model of chronic respiratory diseases; the use of additional cytokines, such as IL-17, to skew differentiation toward an in vitro model of severe chronic respiratory diseases would better reflect the diversity of T2-high and T17-high endotypes and improve the physiological relevance of this in vitro model in future studies. A third limitation of this study was that differences in gene expression were not confirmed at the protein level, raising the potential for unrecognized discordance related to post-transcriptional regulation. Characterizing these differences in gene expression in humans depends on invasive procedures and therefore precludes 

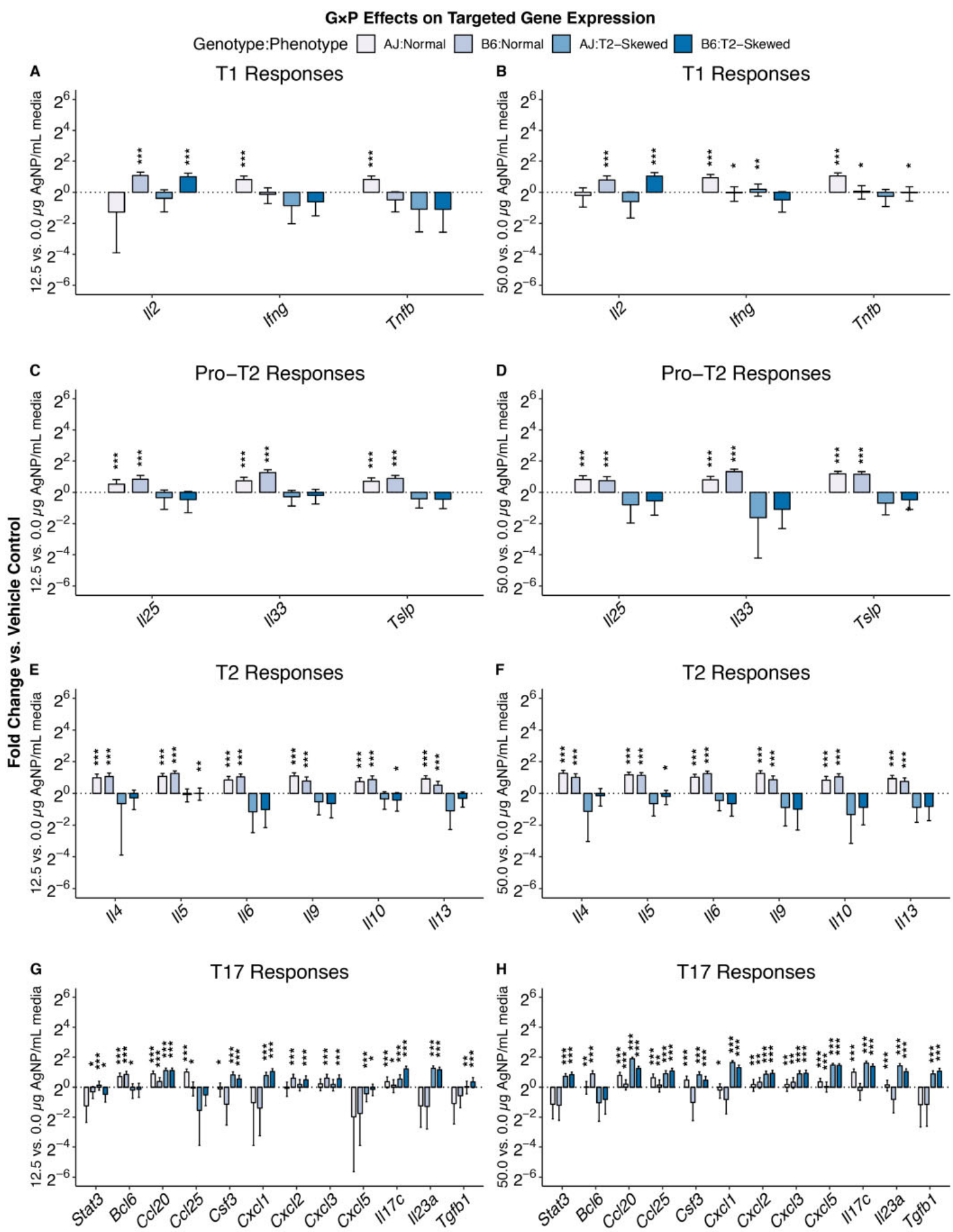

Gene

Figure 7. Genotype, phenotype, and dose effects on targeted gene expression associated with clinical features of severe chronic respiratory diseases. Differential gene expression for T1 responses (A, B), pro-T2 responses (C, D), T2 responses (E, F), and T17 responses (G, H) was characterized using a t test for significant differences in gene expression (FDR $<0.10$ ) on fold changes of $\log _{2}$ (average AgNP/vehicle control) $(n=24)$ for AJ:Normal, B6:Normal, Aj:T2-Skewed, and B6:T2-Skewed exposed to nominal doses of 0, 12.5, or $50 \mu \mathrm{g} \mathrm{AgNP} / \mathrm{ml}$ media for an acute $24 \mathrm{~h}$ exposure. Data represent average $\log _{2}$ (AgNP/vehicle control) fold changes $\pm \mathrm{SE}$; NS ( $\left.p>.05\right)$; ${ }^{*} p<.05$; ${ }^{* *} p<.01 ; * * * 0.001 . n=3$ biological replicates. 


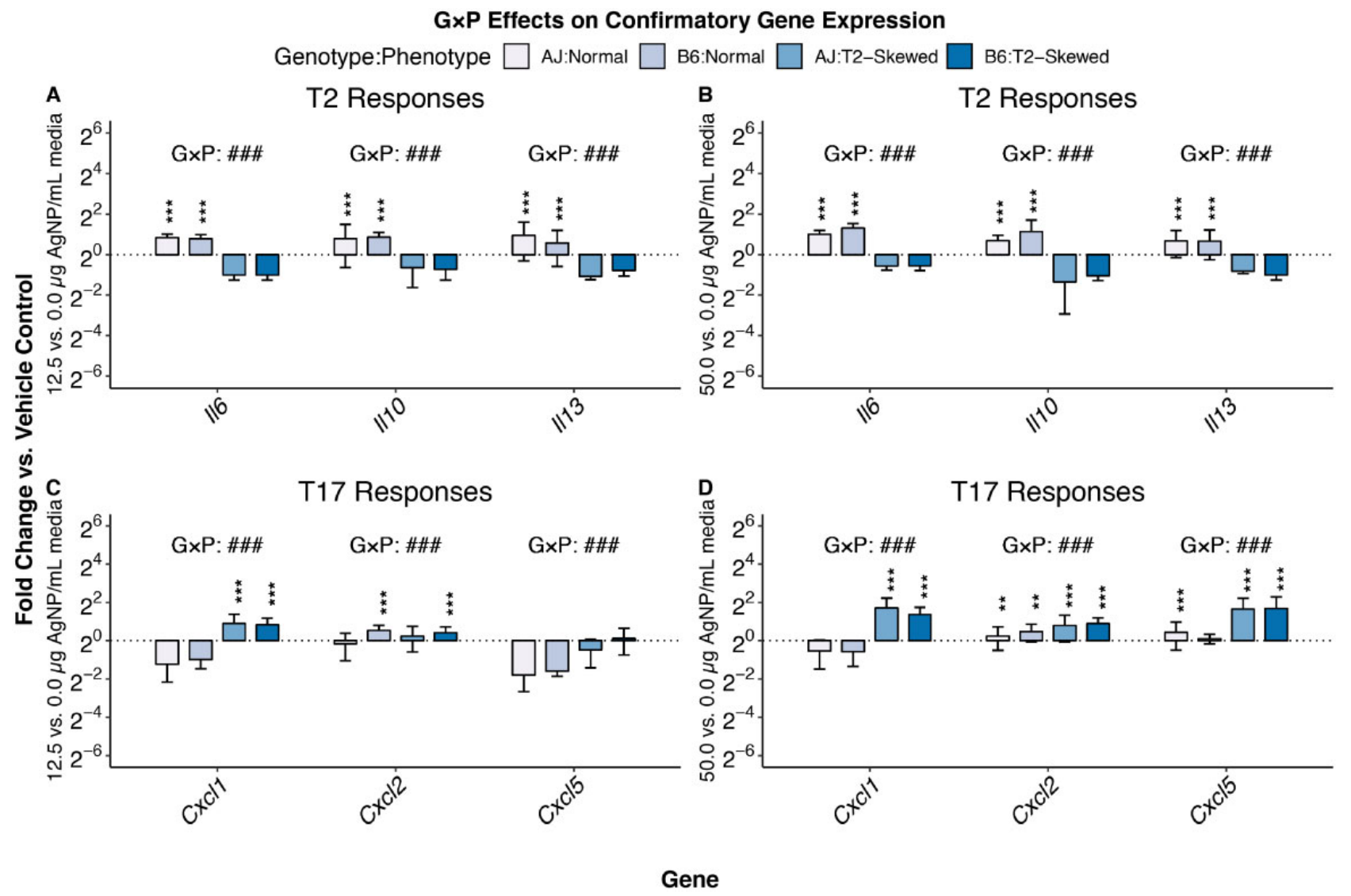

Figure 8. Genotype, phenotype, and dose effects on confirmatory gene expression associated with clinical features of severe chronic respiratory diseases. Total RNA was isolated from organotypic cultures exposed to nominal doses of 0, 12.5, or $50 \mu \mathrm{g} \mathrm{AgNP/ml} \mathrm{media} \mathrm{for} \mathrm{an} \mathrm{acute} 24 \mathrm{~h}$ exposure, and cDNA was subjected to qRT-PCR analysis for confirmatory gene expression of markers associated with (A, B) T2 responses and (C, D) T17 responses. The fold change in gene expression was compared for each genotype and phenotype relative to the vehicle control (asterisks); $\mathrm{G} \times \mathrm{P}$ effects were compared across genotypes and phenotypes (pounds) for each fold change. Data represent mixed effects estimates $\pm 95 \% \mathrm{CI}$; NS $(p>.05) ;{ }^{* *} p<.05 ;{ }^{* * *} p<.001$; \#\#\#p<.001. $n=3$ biological replicates with technical replicates.

routine use, which emphasizes the need to identify less invasive mechanistic-based biomarkers of AgNP toxicity still capable of producing biologically robust predictions of adverse organism responses resulting from these occupational exposures.

Despite these limitations, this study establishes organotypic cultures derived from MTEC as a medium-throughput, highcontent in vitro model of the conducing airway to characterize chemical perturbation as a means to screen and prioritize potential respiratory toxicants. Our results highlight the importance of considering $\mathrm{G} \times \mathrm{P}$ effects on transcriptional regulation when screening and prioritizing potential respiratory toxicants. This is challenging and important for engineered nanomaterials, because their MoA have been shown to differ considerably but are still used in hundreds of consumer products. Prior to anticipating potential adverse organism responses arising from engineered nanomaterial toxicity, ensuring safe development of the consumer products in which they are used will be the most critical and necessary step toward safeguarding public health.

\section{SUPPLEMENTARY DATA}

Supplementary data are available at Toxicological Sciences online.

\section{DECLARATION OF CONFLICTING INTERESTS}

The authors declared no potential conflicts of interest with respect to the research, authorship, and/or publication of this article.

\section{ACKNOWLEDGMENTS}

The authors gratefully acknowledge Dowon An for her technical support with animals, and Yu Hua Chow for her technical support with RNA isolation and purification. Part of this work was conducted at the Genomics Core Laboratory of the Benaroya Research Institute at Virginia Mason.

\section{AUTHOR CONTRIBUTIONS}

TPN, SAG and WAA contributed to experimental design; TPN, AKH performed all cell culture experiments; TPN, TKB, TWW and SAG contributed to RNA-Seq analysis; all authors contributed to writing of the manuscript and approved the final version.

\section{FUNDING}

This work was supported by the United States Environmental Protectional Agency (R835738 to E.M.F., 
T.J.K., W.A.A); National Institutes of Health (P30 ES007033 to T.J.K., T32 ES007032-38 to T.P.N.), and R01 AI137111 to S.A.G.); the Washington State Department of Labor and Industries under the Medical Aid/Accident Fund; (to T.P.N.); and the University of Washington under the Graduate Opportunities for Minority Achievement Program Dissertation Fellowship (to T.P.N.).

\section{REFERENCES}

Ackerman, K. G., Huang, H., Grasemann, H., Puma, C., Singer, J. B., Hill, A. E., Lander, E., Nadeau, J. H., Churchill, G. A., and Drazen, J. M. (2005). Interacting genetic loci cause airway hyperresponsiveness. Physiol. Genomics 21, 105-111.

Alsaleh, N. B., and Brown, J. M. (2018). Immune responses to engineered nanomaterials: Current understanding and challenges. Curr. Opin. Toxicol. 10, 8-14.

Brewer, J. P., Kisselgof, A. B., and Martin, T. R. (1999). Genetic variability in pulmonary physiological, cellular, and antibody responses to antigen in mice. Am. J. Respir. Crit. Care Med. 160, 1150-1156.

Brown, K. R., Krouse, R. Z., Calatroni, A., Visness, C. M., Sivaprasad, U., Kercsmar, C. M., Matsui, E. C., West, J. B., Makhija, M. M., Gill, M. A., et al. (2017). Endotypes of difficultto-control asthma in inner-city African American children. PLoS One 12, e0180778.

Choo, W., Park, C., Jung, S., Moon, B., Ahn, H., Ryu, J., Kim, K.-S., Lee, Y., Yu, I., and Oh, S. (2016). Long-term exposures to low doses of silver nanoparticles enhanced in vitro malignant cell transformation in non-tumorigenic BEAS-2B cells. Toxicol. In Vitro 37, 41-49.

Choy, D. F., Hart, K. M., Borthwick, L. A., Shikotra, A., Nagarkar, D. R., Siddiqui, S., Jia, G., Ohri, C. M., Doran, E., Vannella, K. M., et al. (2015). Th2 and th17 inflammatory pathways are reciprocally regulated in asthma. Sci. Transl. Med. 7, 301ra129.

Choy, D. F., Modrek, B., Abbas, A. R., Kummerfeld, S., Clark, H. F., Wu, L. C., Fedorowicz, G., Modrusan, Z., Fahy, J. V., Woodruff, P. G., et al. (2011). Gene expression patterns of th2 inflammation and intercellular communication in asthmatic airways. J. Immunol. 186, 1861-1869.

Chuang, H.-C., Hsiao, T.-C., Wu, C.-K., Chang, H.-H., Lee, C.-H., Chang, C.-C., and Cheng, T.-J. (2013). Allergenicity and toxicology of inhaled silver nanoparticles in allergenprovocation mice models. Int. J. Nanomed. 8, 4495-4506.

Colobran, R., Pujol-Borrell, R., Armengol, M. P., and Juan, M. (2007). The chemokine network. I. How the genomic organization of chemokines contains clues for deciphering their functional complexity. Clin. Exp. Immunol. 148, 208-217.

Commins, S. P., Borish, L., and Steinke, J. W. (2010). Immunologic messenger molecules: Cytokines, interferons, and chemokines. J. Allergy Clin. Immunol. 125(2 Suppl. 2), S53-72.

Cronholm, P., Karlsson, H. L., Hedberg, J., Lowe, T. A., Winnberg, L., Elihn, K., Wallinder, I., and Möller, L. (2013). Intracellular uptake and toxicity of $\mathrm{Ag}$ and $\mathrm{CuO}$ nanoparticles: A comparison between nanoparticles and their corresponding metal ions. Small 9, 970-982.

Danilczuk, M., Lund, A., Sadlo, J., Yamada, H., and Michalik, J. (2006). Conduction electron spin resonance of small silver particles. Spectrochim. Acta 63, 189-191.

Dobin, A., Davis, C. A., Schlesinger, F., Drenkow, J., Zaleski, C., Jha, S., Batut, P., Chaisson, M., and Gingeras, T. R. (2013). Star: Ultrafast universal RNA-seq aligner. Bioinformatics 29, 15-21.
Gliga, A. R., Skoglund, S., Wallinder, I., Fadeel, B., and Karlsson, H. L. (2014). Size-dependent cytotoxicity of silver nanoparticles in human lung cells: The role of cellular uptake, agglomeration and Ag release. Part. Fibre Toxicol. 11, 11-17.

Hallstrand, T. S., Hackett, T. L., Altemeier, W. A., Matute-Bello, G., Hansbro, P. M., and Knight, D. A. (2014). Airway epithelial regulation of pulmonary immune homeostasis and inflammation. Clin. Immunol. 151, 1-15.

Hammad, H., Chieppa, M., Perros, F., Willart, M. A., Germain, R. N., and Lambrecht, B. N. (2009). House dust mite allergen induces asthma via toll-like receptor 4 triggering of airway structural cells. Nat. Med. 15, 410-416.

Horani, A., Dickinson, J. D., and Brody, S. L. (2013). Applications of mouse airway epithelial cell culture for asthma research. Methods Mol. Biol. 1032, 91-107.

Jang, S., Park, J., Cha, H., Jung, S., Lee, J., Jung, S., Kim, J., Kim, S., Lee, C., and Park, H. (2012). Silver nanoparticles modify VEGF signaling pathway and mucus hypersecretion in allergic airway inflammation. Int. J. Nanomed. 7, 1329-1343.

Jeannet, N., Fierz, M., Kalberer, M., Burtscher, H., and Geiser, M. (2015a). Nano aerosol chamber for in-vitro toxicity (NACIVT) studies. Nanotoxicology 9, 34-42.

Jeannet, N., Fierz, M., Schneider, S., Künzi, L., Baumlin, N., Salathe, M., Burtscher, H., and Geiser, M. (2015b). Acute toxicity of silver and carbon nanoaerosols to normal and cystic fibrosis human bronchial epithelial cells. Nanotoxicology 10, $1-13$

Judson, R. S., Kavlock, R. J., Setzer, R. W., Hubal, E. A., Martin, M. T., Knudsen, T. B., Houck, K. A., Thomas, R. S., Wetmore, B. A., and Dix, D. J. (2011). Estimating toxicity-related biological pathway altering doses for high-throughput chemical risk assessment. Chem. Res. Toxicol. 24, 451-462.

Karp, C. L., Grupe, A., Schadt, E., Ewart, S. L., Keane-Moore, M., Cuomo, P. J., Köhl, J., Wahl, L., Kuperman, D., Germer, S., et al. (2000). Identification of complement factor 5 as a susceptibility locus for experimental allergic asthma. Nat. Immunol. 1, 221-226.

Kim, H., Kim, M., Lee, S., Oh, S., and Chung, K. (2011). Genotoxic effects of silver nanoparticles stimulated by oxidative stress in human normal bronchial epithelial (BEAS-2B) cells. Mutat. Res. 726, 129-135.

Kim, H., Shin, D., Park, Y., Park, C., Oh, S., and Chung, K. (2014). Silver nanoparticles induce p53-mediated apoptosis in human bronchial epithelial (BEAS-2B) cells. J. Toxicol. Sci. 39, 401-412.

Kim, J., Kuk, E., Yu, K., Kim, J.-H., Park, S., Lee, H., Kim, S., Park, Y., Park, Y., Hwang, C.-Y., et al. (2007). Antimicrobial effects of silver nanoparticles. Nanomed. Nanotechnol. Biol. Med. 3, 95-101.

Krewski, D., Andersen, M. E., Mantus, E., and Zeise, L. (2009). Toxicity testing in the 21st century: Implications for human health risk assessment. Risk Anal. 29, 474-479.

Lee, H. S., Park, D. E., Lee, J. W., Chang, Y., Kim, H. Y., Song, W. J., Kang, H. R., Park, H. W., Chang, Y. S., and Cho, S. H. (2017). IL23 secreted by bronchial epithelial cells contributes to allergic sensitization in asthma model: Role of IL-23 secreted by bronchial epithelial cells. Am. J. Physiol. Lung Cell Mol. Physiol. 312, L13-21.

Lee, S., Hwang, H. J., and Kim, Y. (2014). Modeling the role of TGFbeta in regulation of the Th17 phenotype in the LPS-driven immune system. Bull. Math. Biol. 76, 1045-1080.

Lerner, C. A., Sundar, I. K., and Rahman, I. (2016). Mitochondrial redox system, dynamics, and dysfunction in lung inflammaging and COPD. Int. J. Biochem. Cell Biol. 81, 294-306. 
Levitt, R. C., Mitzner, W., and Kleeberger, S. R. (1990). A genetic approach to the study of lung physiology: Understanding biological variability in airway responsiveness. Am. J. Physiol. 258, L157-164.

Ma, R., Levard, C., Marinakos, S. M., Cheng, Y., Liu, J., Michel, M. F., Brown, G. E., and Lowry, G. V. (2012). Size-controlled dissolution of organic-coated silver nanoparticles. Environ. Sci. Technol. 46, 752-759.

Mondal, A., Sawant, D., and Dent, A. L. (2010). Transcriptional repressor BCL6 controls Th17 responses by controlling gene expression in both $\mathrm{T}$ cells and macrophages. J. Immunol. 184, 4123-4132.

Nicholas, T. P., Haick, A. K., Workman, T. W., Griffith, W. C., Nolin, J. D., Kavanagh, T. J., Faustman, E. M., and Altemeier, W. A. 2019. The effects of genotype $x$ phenotype interactions on silver nanoparticle toxicity in organotypic cultures of murine tracheal epithelial cells. BioRxiv 722009.

Park, H., Kim, K., Jang, S., Park, J., Cha, H., Lee, J., Kim, J., Kim, S., Lee, C., Kim, J., et al. (2010). Attenuation of allergic airway inflammation and hyperresponsiveness in a murine model of asthma by silver nanoparticles. Int. J. Nanomed. 5, 505-515.

Quadros, M. E., and Marr, L. C. (2010). Environmental and human health risks of aerosolized silver nanoparticles. J. Air Waste Manage. Assoc. 60, 770-781.

Quadros, M. E., and Marr, L. C. (2011). Silver nanoparticles and total aerosols emitted by nanotechnology-related consumer spray products. Environ. Sci. Technol. 45, 10713-10719.

R Core Team. (2018) R: A Language and Environment for Statistical Computing. $\mathrm{R}$ Foundation for Statistical Computing, Vienna, Austria. https://www.r-bloggers.com/ its-easy-to-cite-and-reference-r/

Schlinkert, P., Casals, E., Boyles, M., Tischler, U., Hornig, E., Tran, N., Zhao, J., Himly, M., Riediker, M., Oostingh, G., et al. (2015). The oxidative potential of differently charged silver and gold nanoparticles on three human lung epithelial cell types. J. Nanobiotechnol. 13, 1.

Scoville, D. K., Botta, D., Galdanes, K., Schmuck, S. C., White, C. C., Stapleton, P. L., Bammler, T. K., MacDonald, J. W., Altemeier, W. A., Hernandez, M., et al. (2017). Genetic determinants of susceptibility to silver nanoparticle-induced acute lung inflammation in mice. FASEB J. 31, 4600-4611.

Scoville, D. K., White, C. C., Botta, D., McConnachie, L. A., Zadworny, M. E., Schmuck, S. C., Hu, X., Gao, X., Yu, J., Dills, R. L., et al. (2015). Susceptibility to quantum dot induced lung inflammation differs widely among the collaborative cross founder mouse strains. Toxicol. Appl. Pharmacol. 289, 240-250.

Seiffert, J., Buckley, A., Leo, B., Martin, N. G., Zhu, J., Dai, R., Hussain, F., Guo, C., Warren, J., Hodgson, A., et al. (2016). Pulmonary effects of inhalation of spark-generated silver nanoparticles in Brown-Norway and Sprague-Dawley rats. Respir. Res. 17, 85.

Seiffert, J., Hussain, F., Wiegman, C., Li, F., Bey, L., Baker, W., Porter, A., Ryan, M. P., Chang, Y., Gow, A., et al. (2015). Pulmonary toxicity of instilled silver nanoparticles: Influence of size, coating and rat strain. PLoS One 10, e0119726.
Shinagawa, K., and Kojima, M. (2003). Mouse model of airway remodeling: Strain differences. Am. J. Respir. Crit. Care Med. 168, 959-967.

Simeone-Penney, M. C., Severgnini, M., Tu, P., Homer, R. J., Mariani, T. J., Cohn, L., and Simon, A. R. (2007). Airway epithelial STAT3 is required for allergic inflammation in a murine model of asthma. J. Immunol. 178, 6191-6199.

Su, C. L., Chen, T. T., Chang, C. C., Chuang, K. J., Wu, C. K., Liu, W. T., Ho, K. F., Lee, K. Y., Ho, S. C., Tseng, H. E., et al. (2013). Comparative proteomics of inhaled silver nanoparticles in healthy and allergen provoked mice. Int. J. Nanomed. 8, 2783-2799.

Suryawanshi, A., Manoharan, I., Hong, Y., Swafford, D., Majumdar, T., Taketo, M. M., Manicassamy, B., Koni, P. A., Thangaraju, M., Sun, Z., et al. (2015). Canonical Wnt signaling in dendritic cells regulates Th1/Th17 responses and suppresses autoimmune neuroinflammation. J. Immunol. 194, 3295-3304.

Vance, M. E., Kuiken, T., Vejerano, E. P., McGinnis, S. P., Hochella, M. F., Rejeski, D., and Hull, M. S. (2015). Nanotechnology in the real world: Redeveloping the nanomaterial consumer products inventory. Beilstein J. Nanotechnol. 6, 1769-1780.

Woodruff, P. G., Modrek, B., Choy, D. F., Jia, G., Abbas, A. R., Ellwanger, A., Koth, L. L., Arron, J. R., and Fahy, J. V. (2009). T-helper type 2-driven inflammation defines major subphenotypes of asthma. Am. J. Respir. Crit. Care Med. 180, 388-395.

Wu, H., Xu, L. L., Teuscher, P., Liu, H., Kaplan, M. H., and Dent, A. L. (2015). An inhibitory role for the transcription factor Stat3 in controlling IL-4 and Bcl6 expression in follicular helper T cells. J. Immunol. 195, 2080-2089.

Yamanaka, K., Fujisawa, T., Kusagaya, H., Mori, K., Niwa, M., Furuhashi, K., Kono, M., Hamada, E., Suda, T., and Maekawa, M. (2018). IL-13 regulates IL-17C expression by suppressing NF-kappaB-mediated transcriptional activation in airway epithelial cells. Biochem. Biophys. Res. Commun. 495, 1534-1540.

Yen, D., Cheung, J., Scheerens, H., Poulet, F., McClanahan, T., McKenzie, B., Kleinschek, M. A., Owyang, A., Mattson, J., Blumenschein, W., et al. (2006). IL-23 is essential for T cellmediated colitis and promotes inflammation via IL-17 and IL-6. J. Clin. Invest. 116, 1310-1316.

You, Y., and Brody, S. L. (2013). Culture and differentiation of mouse tracheal epithelial cells. Methods Mol. Biol. 945, 123-143.

You, Y., Richer, E. J., Huang, T., and Brody, S. L. (2002). Growth and differentiation of mouse tracheal epithelial cells: Selection of a proliferative population. Am. J. Physiol. Lung Cell Mol. Physiol. 283, L1315-1321.

Zhang, H., Wang, X., Wang, M., Li, L., Chang, C., Ji, Z., Xia, T., and Nel, A. E. (2015). Mammalian cells exhibit a range of sensitivities to silver nanoparticles that are partially explicable by variations in antioxidant defense and metallothionein expression. Small 11, 3797-3805. 